\title{
Aeolian Tones Radiated from Flow Over Bluff Bodies
}

\author{
Mohamed Sukri Mat Ali ${ }^{*}$, , Sheikh Ahmad Zaki Shaikh Salim ${ }^{1}$, Mohamad Hafz Ismail ${ }^{1}$, \\ Sallehuddin Muhamad ${ }^{2}$ and Muhammad Iyas Mahzan²
}

\author{
${ }^{1}$ Wind Engineering Laboratory, Malaysia-Japan International Institute of Technology, Universiti Teknologi Malaysia, \\ Kuala Lumpur, Malaysia \\ ${ }^{2}$ Department of Engineering, UTM Razak School of Engineering and Advanced Technology, Universiti Teknologi \\ Malaysia, Kuala Lumpur, Malaysia
}

\begin{abstract}
Bluff body is a simple but a central shape for many engineering applications. The geometry shape of the bluff body characterises the behaviour of the flow over the bluff body, where a more complex flow structure is found near downstream. Shear layer separation is mainly responsible for the periodic global phenomena, that includes the generation of sound. The magnitude of the aerodynamically generated sound is dominated by the fluctuations of aerodynamics forces, i.e., drag and lift. The study also shows that the sound pressure field is shaped by the aeolian tones that is related strongly to the lift fluctuations of the bluff body. Amplitude and frequency of the fluctuating lift change naturally with the shape of a particular bluff body. Triangular cylinder exhibits the largest sound pressure level ( $41.9 \mathrm{~dB})$ followed by ellipse and circular shapes. Square cylinder emits the lowest sound pressure level $(36.7 \mathrm{~dB})$. This corresponds to the longest downstream vortex formation length at which for a square cylinder the long vortex formation length provides space for more vortex to dissipate.
\end{abstract}

Keywords: Bluff body, Passive flow and noise control, Aeolian tones, Shear layer, Vortex shedding.

\section{BACKGROUND OF THE STUDY}

Flow over a bluff body is a fundamental and important subject of fluid mechanics. In the engineering pratical applications, their behaviour in the various flow conditions needs to be well understood and if it is not, the annoying flow-induced-problems may exist and consequently degrade the performance of the design. This can be clearly observed from the noise emitted from a side view mirror of passenger car when moving at high speed, i.e., $80 \mathrm{~km} / \mathrm{h}$ [1], a landing gear system of aircraft when about to land $[2,3]$ and a pantograph system of high speed train when moving at transition speed $\approx 225 \mathrm{~km} / \mathrm{h}$ [4]. The wake generated by the bridge piers is also a concern for civil engineering as it can creates a scour (a hole due to the removal of sediment) around bridge piers when the wake forms a horseshoe vortex in the channel bed [5]. Vibration and strong pressure drag are also of concern for road vehicles and also machinery equipments that contain bluff body parts.

Recently, a series of two dimensional numerical investigations of Ali et al. [6-11] found a mechanism to cancel the noise signal at the far-field from the square cylinder by simply placing a thin flat plate downstream. The theory behind the successful discovery was simple, which was originated from Doolan [12], that was the square cylinder and the plate must generate the same magnitude of

*Address correspondence to this author at the Wind Engineering Laboratory, Malaysia-Japan International Institute of Technology, Universiti Teknologi Malaysia, Kuala Lumpur, Malaysia; Tel: +603 - 2203 1286; Fax: +603 - 2203 1266; E-mail: sukri@ic.utm.my lift fluctuations but their signals must be out-of-phase. However, to achieve this condition was not as simple as the theory. This was due to the strong flow interaction and feedback signal between the square cylinder and the plate. Therefore, their numerical simulation could not achieve a total sound cancellation at the far-field even though a significant noise reduction was obtained. Flow visualisation showed that the downstream plate experienced a stall that had distorted the sinusoidal pattern of the lift fluctuations of the plate when compared to the lift fluctuation of the square cylinder.

Therefore, an extension study is required to explore this passive flow and noise control for bluff body with different shapes. There is no available study, at least to the author's knowledge, to investigate the possibility of a downstream flat plate to be made as an anti noise source signal, i.e. lift fluctuations, except for a square cylinder case. Therefore, this study provides the necessary physical flow and noise features for flow over an isolated bluff body in four different geometrical shapes. This information is vital in designing a downstream flat plate for a sound cancellation in the later stage.

\section{PROBLEM SETUP}

The flow specification is set similar to the previous investigations of Ali et al. [6-11]. However, this continuation study investigates four different bluff body shapes, i.e.; Triangle, Ellipse, Circular and Square. Fig. (1) shows a schematic diagram of the problem geometry. The streamwise and cross-stream wise lengths of the bluff bodies for all cases are made the same, $D$. The Reynolds number based on 
the free stream velocity and a length scale $D$, $R e=U_{\infty} D / v$, is 150 . The Mach number chosen for this study is $M=0.2$.

\section{NUMERICAL SIMULATION}

\subsection{Flow Simulations}

The flow is modelled in two-dimensional governing equations to reduce the computational cost. This assumption is valid by the fact that the Reynolds number of the current study is lower than the critical Reynolds number for the onset of three-dimensional instability, which is $R e=161$ according to the Floquet stability analysis of Robichaux et al. [13]. Numerical investigations of Saha et al. [14], Blackburn and Lopez [15] and experimental investigations of Luo et al. [16, 17] also confirmed that the flow over a bluff body became three-dimensional when the Reynolds number was $R e>160$. Additionally, Sohankar et al. [18] also agreed that there was no difference between two- and three-dimensional simulations of flow around a single square cylinder at $R e=150$.

The governing equation is based on the two-dimensional incompressible Navier-Stokes equations:

$$
\begin{aligned}
& \frac{\partial u_{\mathrm{i}}}{\partial t}+u_{\mathrm{j}} \frac{\partial u_{\mathrm{i}}}{\partial x_{\mathrm{j}}}=-\frac{1}{\rho_{0}} \frac{\partial p}{\partial x_{\mathrm{i}}}+\frac{\partial}{\partial x_{\mathrm{j}}}\left[v\left(\frac{\partial u_{\mathrm{i}}}{\partial x_{\mathrm{j}}}+\frac{\partial u_{\mathrm{j}}}{\partial x_{\mathrm{i}}}\right)\right] \\
& \frac{\partial u_{\mathrm{i}}}{\partial x_{\mathrm{i}}}=0
\end{aligned}
$$

where subscripts $\mathrm{i}, \mathrm{j}=1,2$ refer to the axis in the Cartesian coordinate system, i.e., streamwise $(x)$ and crossstream $(y)$ directions, respectively. The origin of the Cartesian coordinates is the centre of the square cylinder. The velocity $u$ and pressure $p$ are defined in a collocated manner in the computational domain and PISO algorithm [19] is used to solve the pressure-velocity coupling. The partial differential equation is discretised based on the finite volume method using OpenFOAM [20] numerical simulation system.

The grid solution for the current study is similar to the finest grid solution used by the extensive grid refinement study of Ali et al. [21]. Readers can refer to the reference for the detailed solution of the numerical simulation. Table $\mathbf{1}$

shows a good agreement between the results from the high grid resolution of direct numerical simulation (DNS) of Ali et al. [21] with other similar investigations.

Table 1. Comparison of the Finest Grid Resolution of [21] with Other Similar Previous Studies (Square Cylinder)

\begin{tabular}{|c|c|c|c|}
\hline Previous Studies & $C_{L_{\mathrm{rms}}}$ & $C_{D_{\text {mean }}}$ & $S t$ \\
\hline \hline Experiments [18, 22] & - & 1.40 & 0.148 to 0.155 \\
\hline DNS of Doolan [12] & 0.296 & 1.44 & 0.156 \\
\hline DNS of Sohankar et al. [23] & 0.230 & 1.44 & 0.165 \\
\hline DNS of Inoue et al. [24] & $\begin{array}{c}0.40 \\
\text { (peak) }\end{array}$ & 1.40 & 0.151 \\
\hline DNS of Ali et al. [21] & 0.285 & 1.47 & 0.160 \\
\hline
\end{tabular}

\subsection{Acoustic Simulation}

The sound sources are obtained from the direct numerical simulation of the flow (near field) as described in Sec. 3.1. These sound sources are then used in the Lighthill's acoustic analogy [25] to evaluate the level and character of the sound radiation. Lighthill's acoustic analogy is actually derived from the rearrangement of the Navier-Stoke's equations. Therefore, it is an exact equation for physics of sound generation and propagation.

Using the time derivative of the continuity equation and also taking the divergence of the momentum equation, then subtracting the two equations, the sound propagation can be written in an inhomogeneous wave equation;

$$
\begin{aligned}
& \left(\frac{1}{c_{0}^{2}} \frac{\partial^{2}}{\partial t^{2}}-\nabla^{2}\right)\left(c_{0}^{2}\left(\rho-\rho_{0}\right)\right)=\frac{\partial^{2} T_{\mathrm{ij}}}{\partial x_{\mathrm{i}} \partial x_{\mathrm{j}}} \\
& T_{\mathrm{ij}}=\rho u_{\mathrm{i}} u_{\mathrm{j}}-\tau_{\mathrm{ij}}+\delta_{\mathrm{ij}}\left(\left(p-p_{0}\right)-c_{0}^{2}\left(\rho-\rho_{0}\right)\right)
\end{aligned}
$$

Lighthill's stress tensor $\left(T_{\mathrm{ij}}\right)$ accounts for sound generation due to the Reynolds stress tensor $\left(u_{\mathrm{i}} u_{\mathrm{j}}\right)$, viscous stresses $\left(\tau_{\mathrm{ij}}\right)$ and also entropy fluctuations ( $\delta_{\mathrm{ij}}\left(\left(p-p_{0}\right)-c_{0}^{2}\left(\rho-\rho_{0}\right)\right)$, where $\delta_{\mathrm{ij}}$ is the Kronecker delta).

To obtain an integral equation which includes the effects
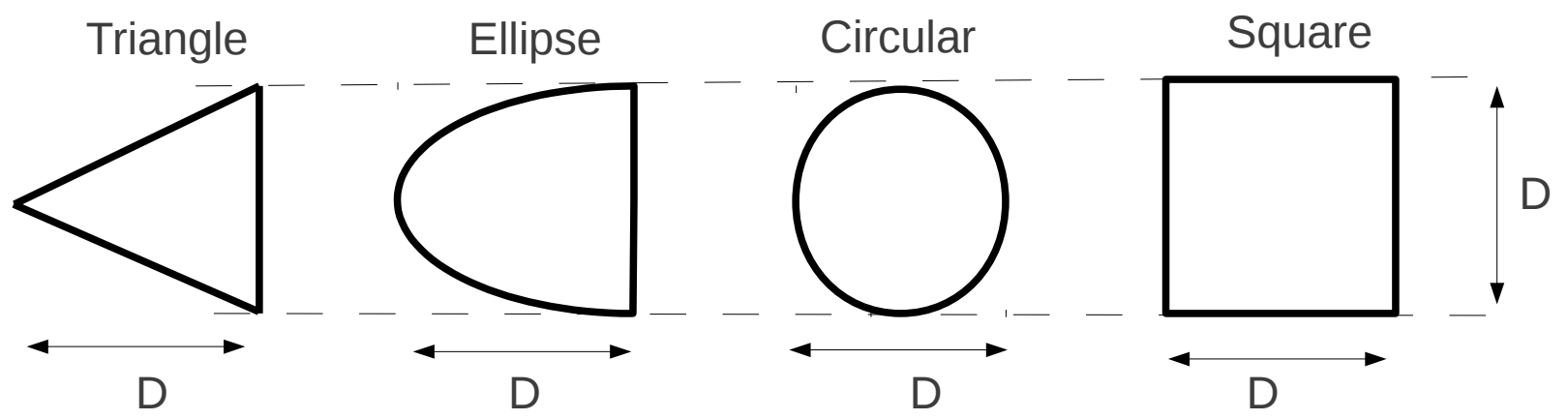

Fig. (1). Four different bluff body shapes under investigation. 
of sound sources, boundary condition of the sound field and its initial condition for solving Eq. (3), a Green's function $(G)$ is employed;

$$
\left(\frac{1}{c_{0}^{2}} \frac{\partial^{2}}{\partial t^{2}}-\nabla^{2}\right) G\left(x, y, t-t^{\prime}\right)=\delta(x-y) \delta\left(t-t^{\prime}\right)
$$

The Green's function (Eq. (5)) defines the response of the flow at observer's position $(x)$ to an impulsive source at position $y$. The observer receives the response at time $t$ when the source is released at time $t^{\prime}$. The Dirac delta function $(\delta)$ is the causality for the Green's function, $G\left(x, y, t-t^{\prime}\right)=0$.

To include the effect of solid surface in the generation of sound, Curle solution [26] for Lighthill acoustic analogy is applied. The solution of Lighthill's equation can be rewritten to include the effect of surface $S$;

$c_{0}^{2}\left[\rho(x, t)-\rho_{0}\right]=\frac{\partial^{2}}{\partial x_{\mathrm{i}} \partial x_{\mathrm{j}}} \int_{V} d y \int_{-\infty}^{\infty} T_{\mathrm{ij}}\left(y, t^{\prime}\right) G\left(x, y, t-t^{\prime}\right) d t^{\prime}$

$+\frac{\partial}{\partial x_{\mathrm{i}}} \int_{S} d S_{\mathrm{j}}(y) \int_{-\infty}^{\infty} p_{\mathrm{ij}}^{\prime}\left(y, t^{\prime}\right) G\left(x, y, t-t^{\prime}\right) d t^{\prime}$.

The additional term in the Lighthill solution is the sound generation due to the solid surface that experiences a compressive stress $\left(p_{\mathrm{ij}}^{\prime}=\left(p-p_{0}\right) \delta_{\mathrm{ij}}-\tau_{\mathrm{ij}}\right)$.

Green's function in two-dimensional space is applied to find the integral solution of Eq. 6;

$$
G\left(x, y, t-t^{\prime}\right)=\frac{H\left[\left(t-t^{\prime}\right)-r / c_{0}\right]}{2 \pi \sqrt{\left(t-t^{\prime}\right)^{2}-r^{2} / c_{0}^{2}}}
$$

where the response of the flow to an impulsive point source is governed by the Heaviside step function $(\mathbf{H})$ which is unity when the time taken for the impulsive point source to travel to the observer is longer than the sound signal to travel at the same distance, $\left[\left(t-t^{\prime}\right)-r / c_{0}\right]>0$ and zero when it takes less than the time taken for the sound signal to travel at the same distance, $\left[\left(t-t^{\prime}\right)-r / c_{0}\right]<0$. The distance between the point source and observer location is $r=|x-y|$ and $c_{0}$ is the speed of sound.

Following the steps and notations of Inoue and Hatakeyama [27], the two-dimensional Curle's solution for equation (6) can be re-written as:

$$
\begin{aligned}
& c_{0}^{2}\left[\rho(x, t)-\rho_{0}\right]=\frac{\partial}{\partial x_{\mathrm{i}} \partial x_{\mathrm{j}}} \int_{V} d y \int_{-\infty}^{t-r / c_{0}} \frac{T_{\mathrm{ij}}\left(y, t^{\prime}\right) d t^{\prime}}{2 \pi \sqrt{\left(t-t^{\prime}\right)^{2}-r^{2} / c_{0}^{2}}} \\
& -\frac{\partial}{\partial x_{\mathrm{i}}} \oint_{s} d l(y) \int_{-\infty}^{t-r / c_{0}} \frac{f_{\mathrm{i}}\left(y, t^{\prime}\right) d t^{\prime}}{2 \pi \sqrt{\left(t-t^{\prime}\right)^{2}-r^{2} / c_{0}^{2}}} .
\end{aligned}
$$

The compressive stress is now represented by the fluctuating of forces per unit length that is acting on the rigid body, $f_{\mathrm{i}} d l(y)=-p_{\mathrm{ij}}^{\prime} d S_{\mathrm{j}}(y)$. Our previous study [9] has shown that for low Reynolds and Mach numbers, the sound radiation is dominated by the sound source from the fluctuating lift and the contribution from the Lighhill's stress tensor components to the total sound level is insignificant. This is also supported by the argument of Inoue and Hatakeyama [27]. They estimated that for two-dimensional sound radiation, the sound pressure levels for different types of sound sources are varied by $p_{\mathrm{q}}^{\prime} / \rho U_{\infty}^{2} \propto A M^{7 / 2} / r^{1 / 2}$ for dipole and $p_{\mathrm{d}}^{\prime} / \rho U_{\infty}^{2} \propto M^{5 / 2} / r^{1 / 2}$ for quadrupole types of sound radiation.

The final form of the two-dimensional Curle's equation (Eq. (8)) for the current study is:

$$
p^{\prime}(x, t)=\frac{x_{\mathrm{i}}}{2^{3 / 2} \pi c_{0}^{1 / 2} r^{3 / 2}} \int_{-\infty}^{\tau}\left[\frac{\partial F_{\mathrm{i}}}{\partial t^{\prime}}\right] \frac{d t^{\prime}}{\sqrt{\tau-t^{\prime}}} .
$$

The time gradient of lift fluctuations $\left(\frac{\partial F}{\partial t^{\prime}}\right)$ is obtained from the direct numerical simulation of the near field and $\tau=t-r / c_{0}$ is the retarded time.

\section{RESULTS}

\subsection{Key Results}

Table 3 shows the key results obtained from the current study. Square cylinder exhibits the lowest magnitude for all parameters being assessed, followed by circular and ellipse, while the triangular shape is the highest (except for Strouhal number). This suggests that passive flow and noise controls are increasingly important when the bluff bodies are modified from square to triangular shape. However, before a secondary body is introduced into the system for a passive flow and noise controls, it is important to understand the fundamental physics aspect of flow over an isolated bluff body. The next sections of the paper discuss in details the physics of fluids and noise generation on four basic geometry types of bluff bodies.

Table 2. Previous Study for a Square Cylinder with a Downstream Flat Plat at $R e=150$

\begin{tabular}{|c|c|c|c|c|c|c|}
\hline Reference & $L$ & $G$ & $S t$ & $C_{L_{\text {rms }}}$ & $C_{D_{\text {mean }}}$ & $\tilde{P}_{\text {rms }}$ \\
\hline \hline Ali et al. $[7-9]$ & $D$ & 0 & 0.145 & 0.225 & 1.336 & $33.98 d B$ \\
\hline Ali et al. $[6,10,11]$ & $D$ & $2.3 D$ & 0.116 & 0.047 & 0.301 & $35.57 d B$ \\
\hline Ali et al. $[10,11]$ & $0.26 D$ & $5.6 D$ & 0.158 & 0.289 & 1.460 & $30.41 d B$ \\
\hline
\end{tabular}


Table 3. Key Results

\begin{tabular}{|c|c|c|c|c|}
\hline Case & $S t$ & $C_{L_{\text {rms }}}$ & $C_{D_{\text {mean }}}$ & $\tilde{P}_{\text {rms }}$ \\
\hline \hline Triangle & 0.2143 & 0.4447 & 1.883 & $41.90 d B$ \\
\hline Ellipse & 0.2180 & 0.3927 & 1.474 & $40.77 d B$ \\
\hline Circular & 0.1880 & 0.3825 & 1.368 & $40.03 d B$ \\
\hline Square & 0.1600 & 0.2850 & 1.470 & $36.71 d B$ \\
\hline
\end{tabular}

\subsection{Flow Field}

The unsteady flow is responsible for the aerodynamically generated sound [28-30]. The current study satisfies the similar flow behaviour. Fig. (1) shows the flow structures in terms of instantaneous spanwise vorticity contours and velocity vector at time when the lift of the body is maximum. Kármán vortex street is clearly observed downstream for all cases. This von Kármán vortex is generated periodically from the shear layers that is separated from the body before they roll-up immediately downstream. The rolling-up shear layers are more close to the body for a triangle when compared to the square cylinder.

The vortex shedding frequency is strongly related to the length required for the von Kármán vortex to form fully. This strong and large vortex is originated from the shear layer that is separated on the side surfaces of the bluff body [8, 31]. A staggered arrangement of von Kármán vortex is observed for all cases being investigated. However, triangle and ellipse cylinders exhibit a shorter vortex formation length than that for circular and square cylinders. This is

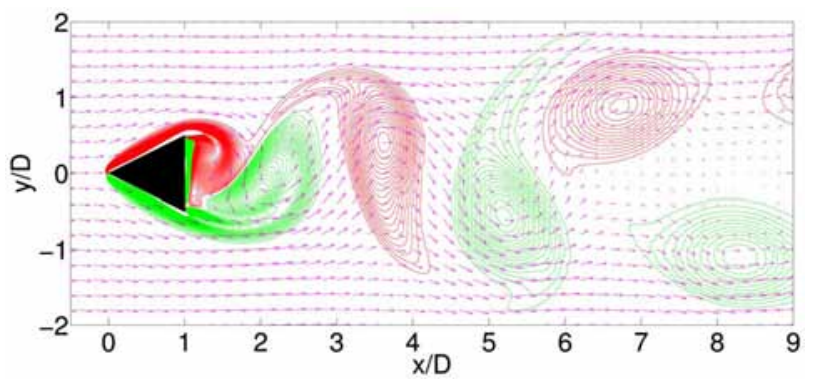

(a) Triangle

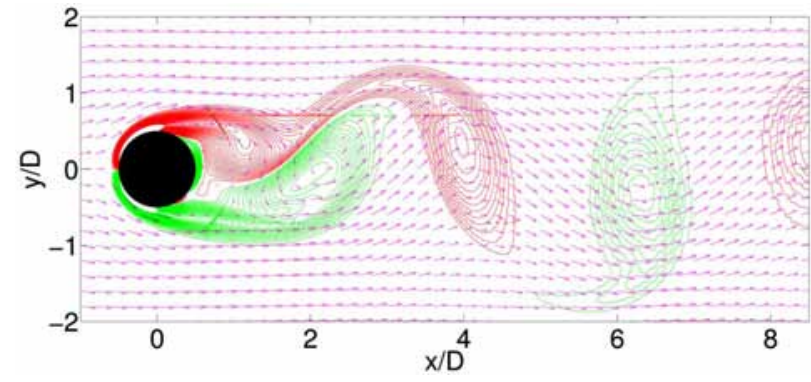

(c) Circular clearly shown in Fig. (2a-d). The shorter vortex formation length increases the rate of vortex formation that consequently increases the Strouhal number. The vortex formation also requires entrainment of irrotational flow, i.e. free-stream, to grow fully. This entrainment is assisted by the opposite sign of the growing vortex. Cylinder with larger wake width take a longer time for the growing vortex to form fully due to the distance between the two opposite vortices that are far apart [8, 32-34]. This is what happens to the triangle cylinder, where the distance between the two opposite vortices is larger than for the case of ellipse (compare Fig. (2a) and (2b)). Similar argument is also used for the Strouhal number reduction between the circular and square cylinders.

The vortex formation process attributes to the highly concentrated streamwise velocity fluctuations, $U_{x x}$ [10]. This is shown in Fig. (3). The point of concentrations is located just outside of the recirculation region, i.e. bubble, marked with dotted line. High magnitude of $U x x$ is responsible for high magnitude in lift and drag. A compact vortex that is formed downstream of the triangle cylinder instigates high velocity fluctuation at that region. This explains the results obtained in Table 3 .

Fig. (4) shows time histories of aerodynamic forces for all cases. A sinusoidal form is observed for lift and drag forces in all cases being investigated. Previous studies by Doolan [12] and Ali et al. [11] have shown that this form of fluctuating forces is suitable for passive sound cancellation mechanism using a downstream flat plate. This suggests that the same method of passive sound cancellation mechanism can also be applied for different geometry of bluff body. Additionally, the frequency of the drag signal is twice that of

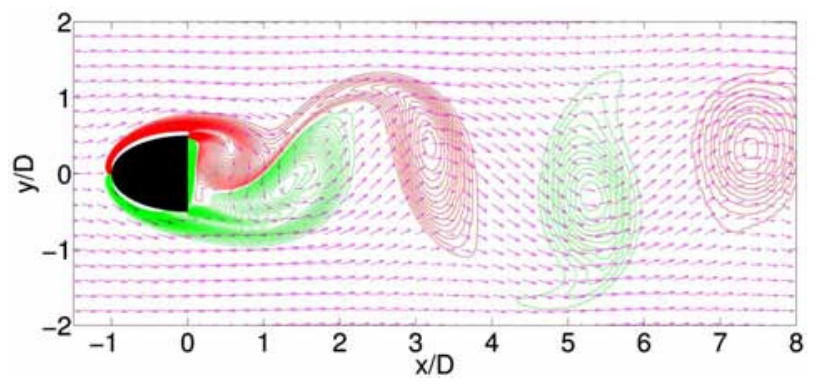

(b) Ellipse

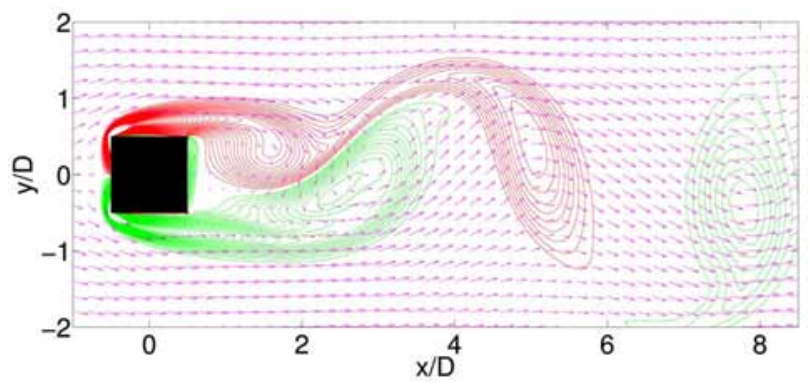

(d) Square

Fig. (2). Contours of instantaneous spanwise vorticity when the lift at the cylinder is maximum. 


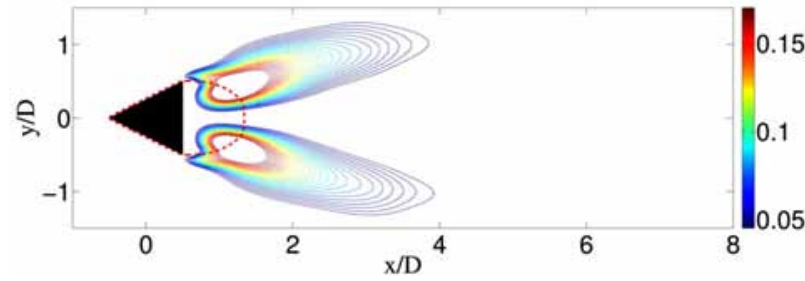

(a) Triangle

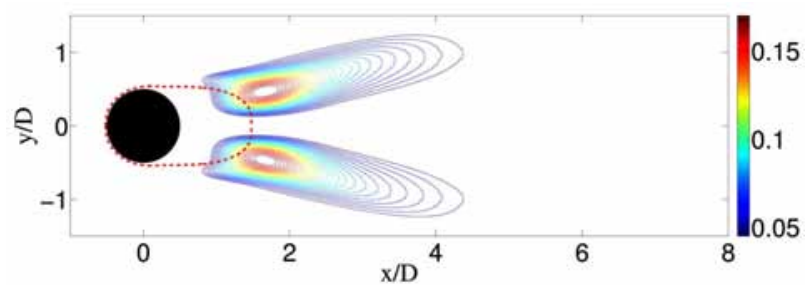

(c) Circular

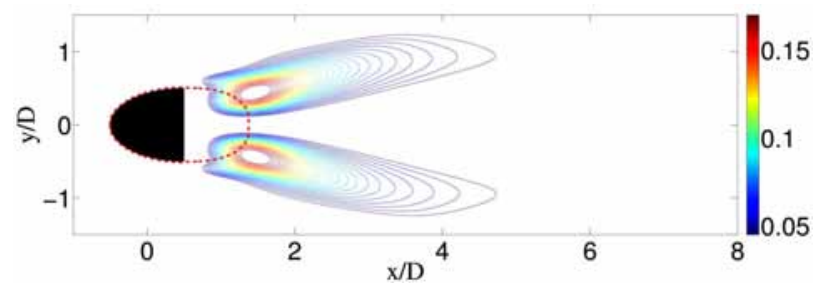

(b) Ellipse

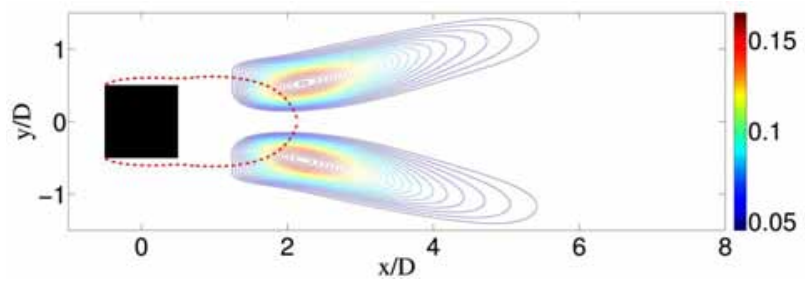

(d) Square

Fig. (3). Contours of time-averaged streamwise normal stress. The dotted line indicating the recirculation region.

the lift signal, identical to the results obtained for the case of bluff body in a tandem arrangement [35], semi-circular cylinder with a splitter plate [36] and circular cylinder with slip splitter plate [37]. This can simply be explained by counting the number of shear layer engulfs on the surfaces of the cylinder, i.e., back surface (mainly responsible for drag) has two shear layers while at top and bottom surfaces (mainly responsible for lift) has only one shear layer.

Power spectrum density analyses for the lift and drag indicate a strong tonal signal at the frequency of the vortex shedding for the lift and twice of the frequency of the vortex shedding for the drag. This is shown in Figs. $(\mathbf{5}, \mathbf{6})$, respectively. An additional series of tones is observed for lift and drag signals in the case of ellipse cylinder. The reason for these additional tones is still not clearly understood . However, studies by Gerrard [31] and Unal and Rockwell [38], for the case of an isolated circular cylinder, showed that it was possible for the fluctuating flow properties to exhibit series of additional tones. These additional tones were generated by the signal from the small scale vortices that were embedded inside the shear layers. This vortex was known as Bloor-Gerrard vortex, where it was initiated from the Kelvin-Helmholtz instability.

\subsection{Acoustic Field}

The sound directivities that are obtained using Eq. (9) of Curle acoustics solution are compared with the results of Inoue and Hatakeyama [27] who directly calculated the

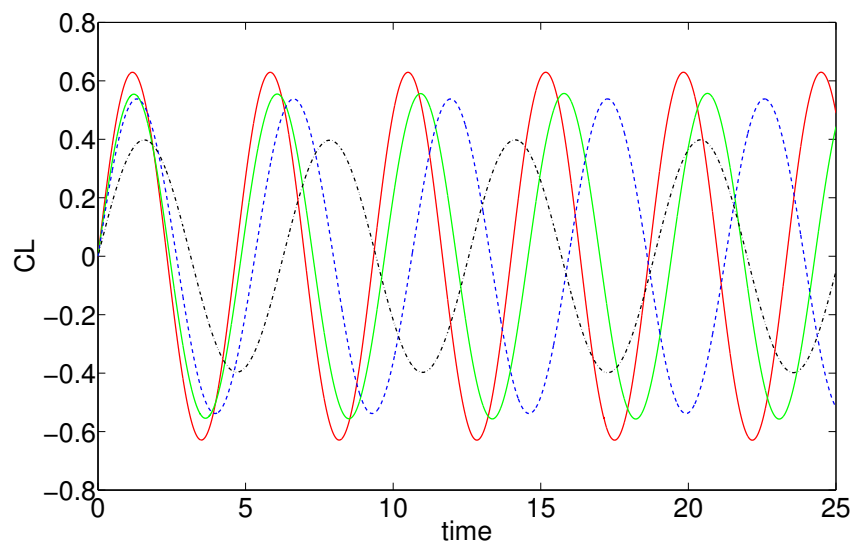

(a) Lift

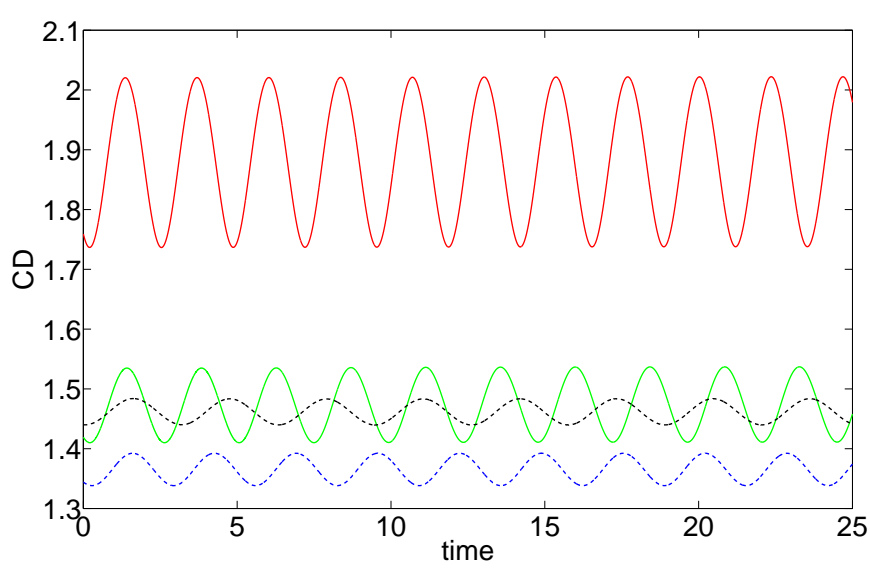

(b) Drag

Fig. (4). Fluctuations of lift, (a) and drag, (b). Triangle; red-solid line, Ellipse; green-solid line, Circular; black-broken line and Square; bluebroken line. 


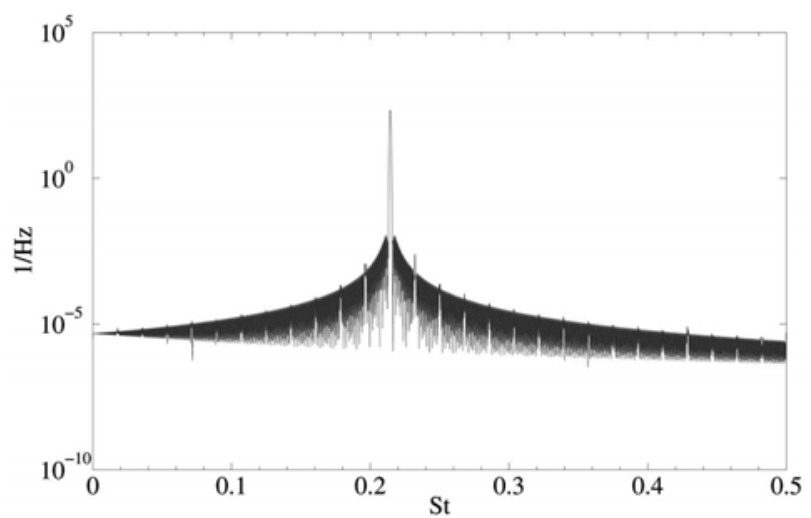

(a) Triangle

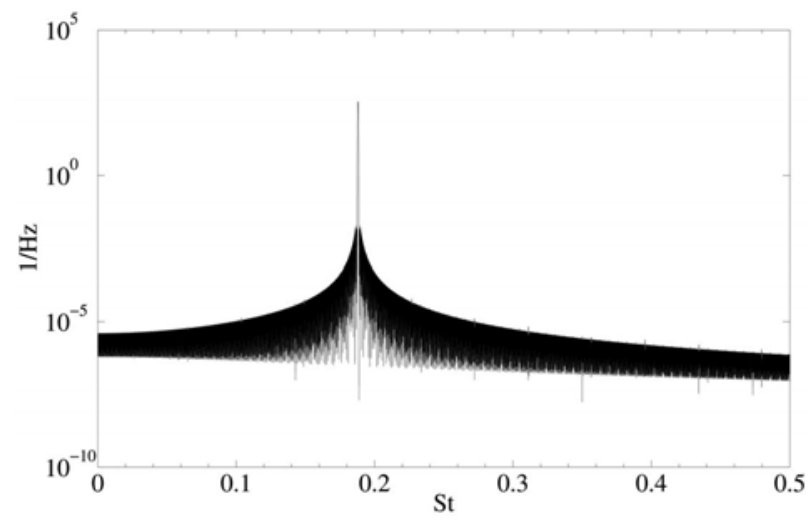

(c) Circular

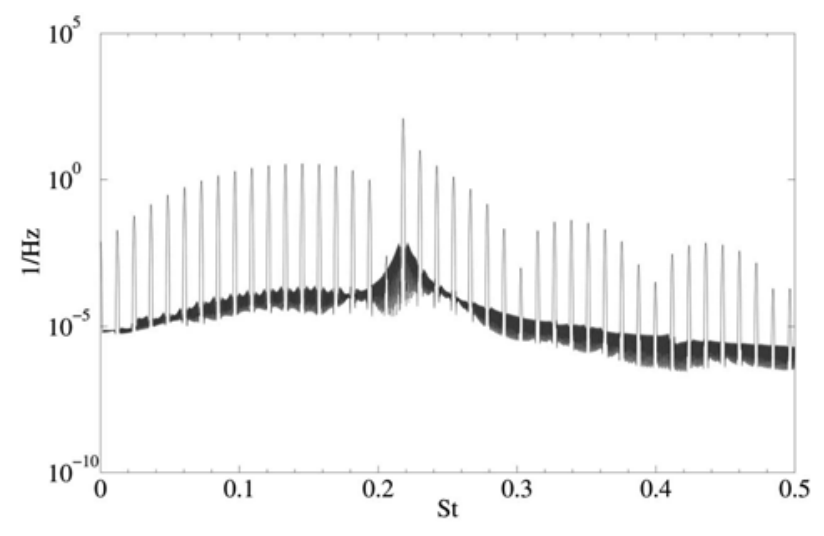

(b) Ellipse

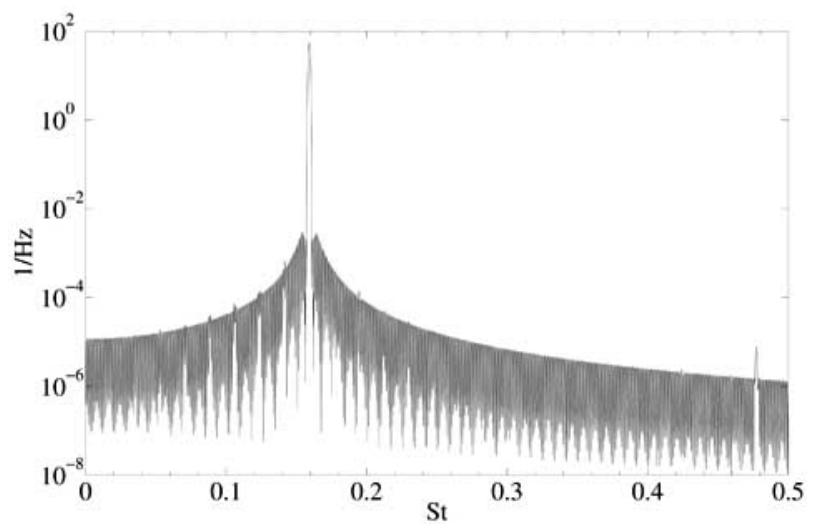

(d) Square

Fig. (5). Power spectrum density for the lift fluctuation.

sound radiation using compressible DNS analysis (see Fig 7). Even though the current study only takes into account the sound source from the fluctuating lift, identical results are obtained for the both studies. This is in agreement with the observations of acoustic analyses by Inoue and Hatakeyama [27], Inoue et al. [24] and Inoue et al. [39]. They observed that the strong aeolian tone radiated from a bluff body is associated with the periodic shedding of von Kármán vortex, where this kind of flow phenomenon is responsible for inducing the fluctuating lift on the body. This concludes that the dominant sound source for all cases is the lift fluctuation.

The sound sources that are emitted due to the lift and drag are plotted in Figs. $(\mathbf{8}, \mathbf{9})$, respectively. The sound pressure level slowly decreases with the distance from the sound source. For sound field that is generated by the lift, the sound waves radiates at the direction $\pm 90^{\circ}$ from the freestream. Contrary, for sound field that is generated by the drag, the sound waves radiates in the direction $\pm 0^{\circ}$ from the free stream. But, both sound sources are being transmitted in a similar pattern, i.e. dipole, except that the peaks follow the direction of their respective forces. This is consistent with the argument of Curle [26] and agrees well with the dimensional analysis of Inoue and Hatakeyama [27].

Fig. (10a, b) compare the magnitude of root mean square (rms) sound pressure ( $\left.\tilde{P}=p_{\text {rms }}^{\prime} / \rho U_{\infty}^{2}\right)$, that is emitted due to the lift and drag fluctuations, respectively, for all cases being investigated. The increase in the magnitude of $\tilde{P}$ corresponds to the increase in the magnitude of aerodynamic forces. For all cases, the magnitude of $\tilde{P}$ for the sound source from the drag signal is small, one order of magnitude lower than the magnitude of $\tilde{P}$ from the lift signal. The reason is the small amplitude of drag fluctuation if compared with the amplitude of lift fluctuations.

The time histories of the sound pressure level for both sound sources, i.e. lift and drag, also have the sinusoidal pattern similar to the fluctuating aerodynamic forces. See Fig. (11). This information is important if one wants to apply a downstream flat plate for a sound cancellation mechanism. For total sound cancellation, a downstream flat plate must be able to imitate the sound pressure fluctuation from the bluff body with the same magnitude but the signal must be out of 


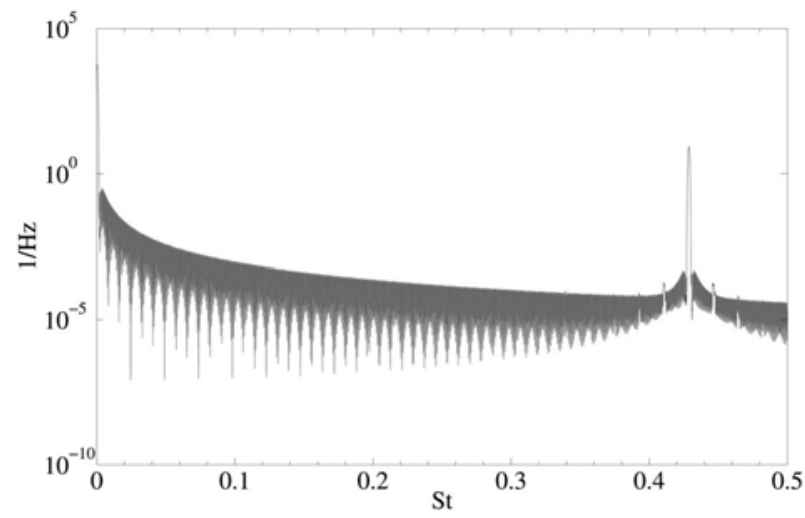

(a) Triangle

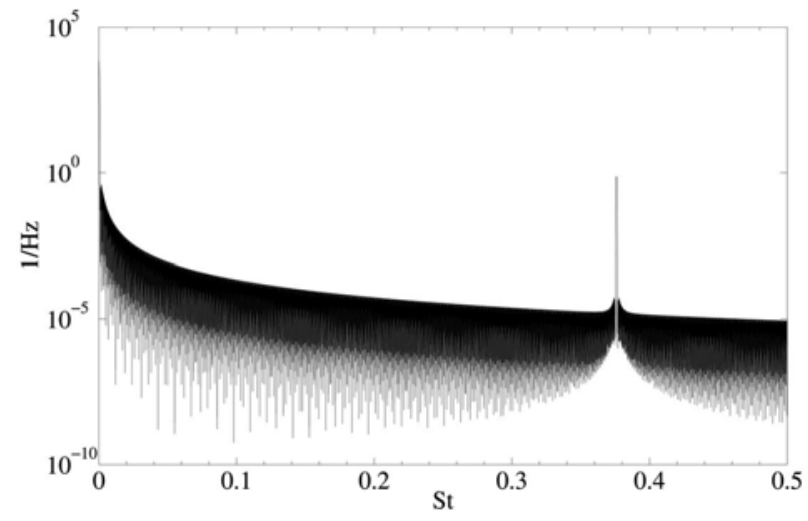

(c) Circular

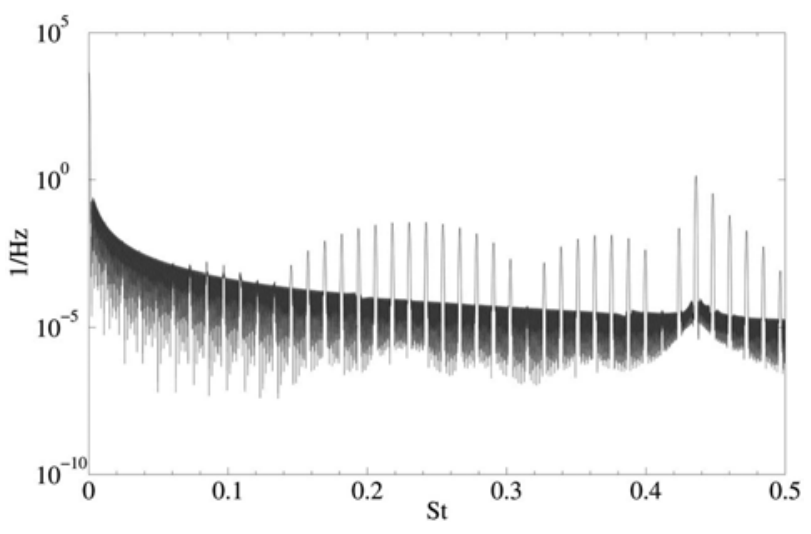

(b) Ellipse

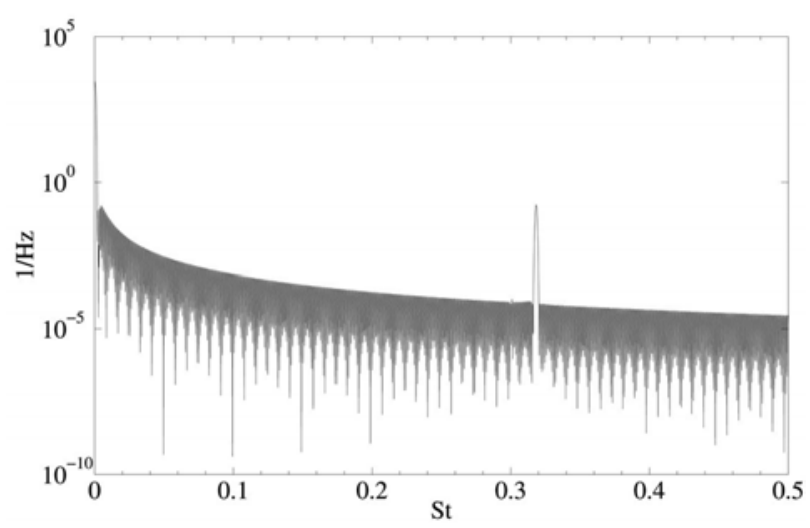

(d) Square

Fig. (6). Power spectrum density for drag fluctuation.

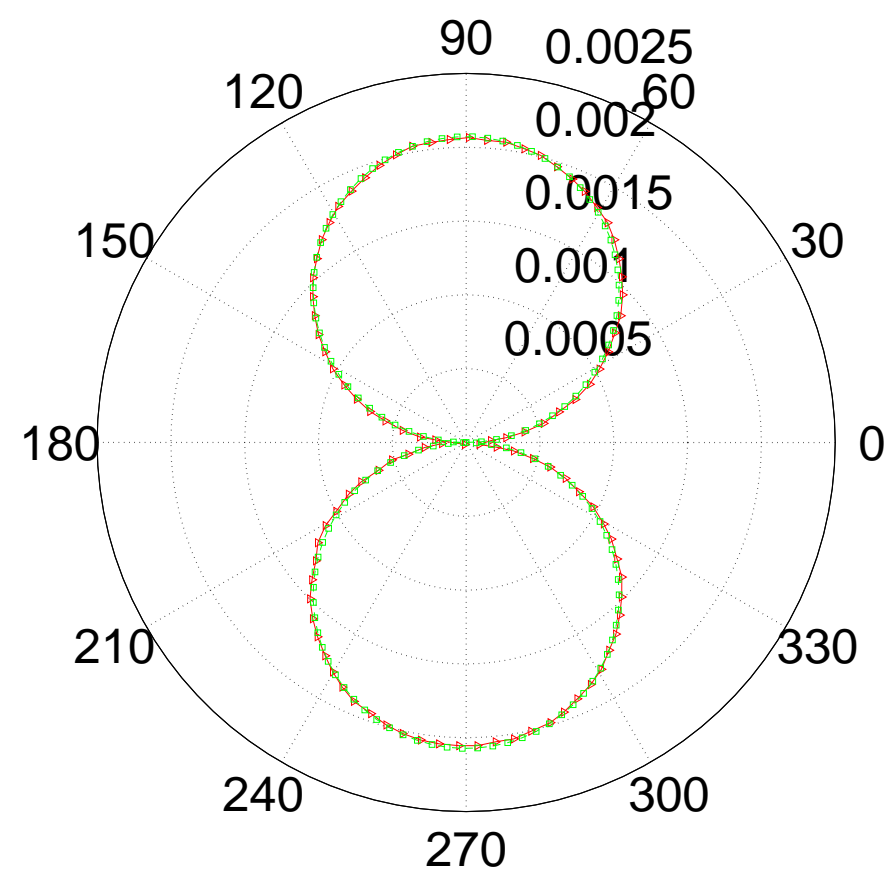

Fig. (7). Sound directivity comparison between current study and DNS of Inoue and Hatakeyama [27] for a circular cylinder at $R=75 D$. 


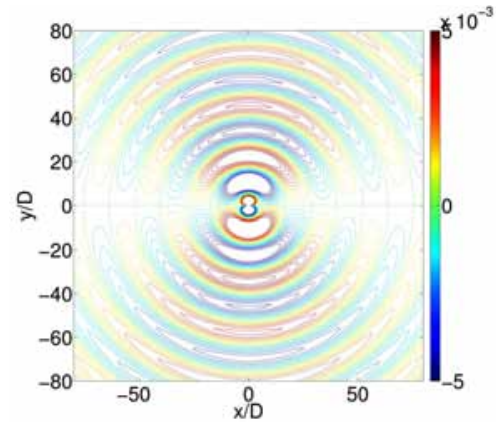

(a) Triangle

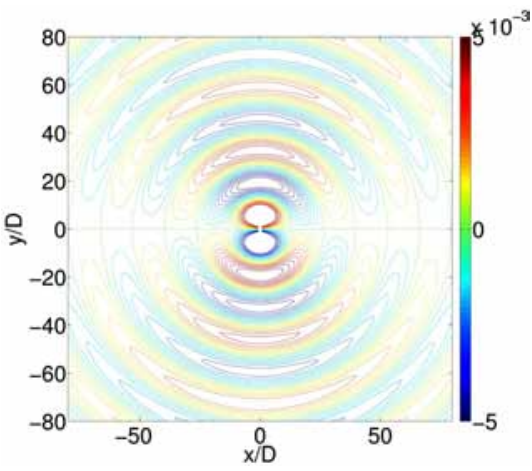

(c) Circular

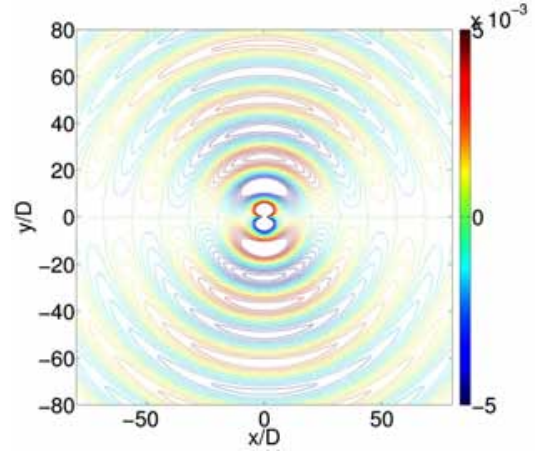

(b) Ellipse

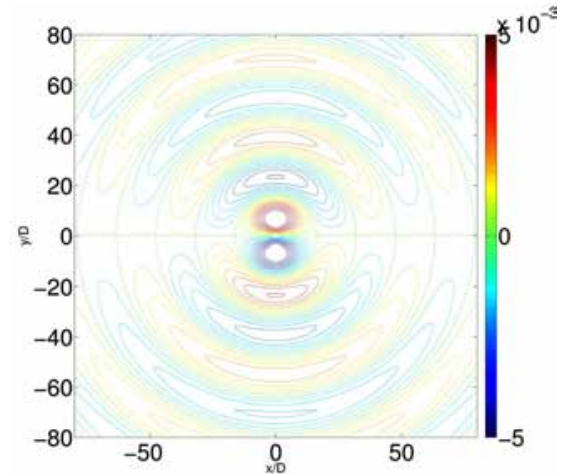

(d) Square

Fig. (8). Contours of sound pressure level due to the fluctuation of lift.

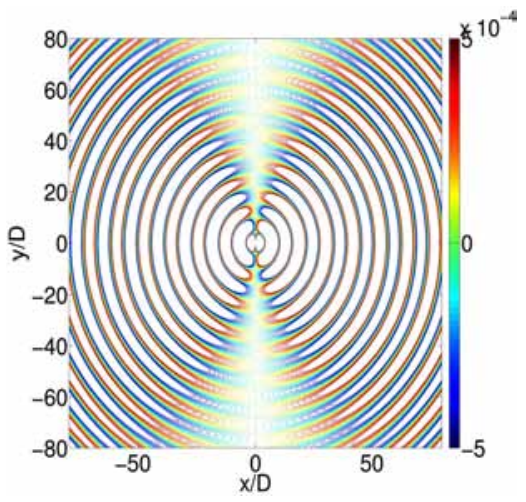

(a) Triangle

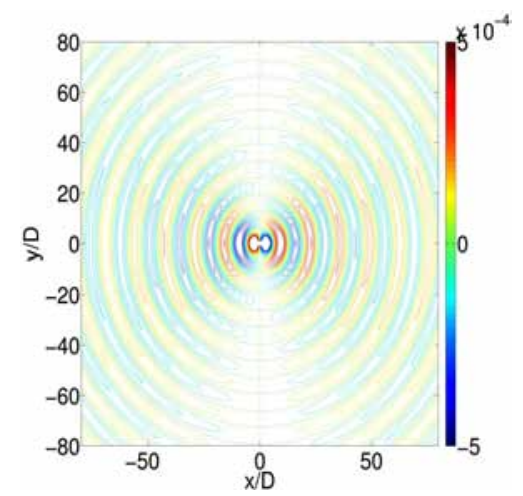

(c) Circular

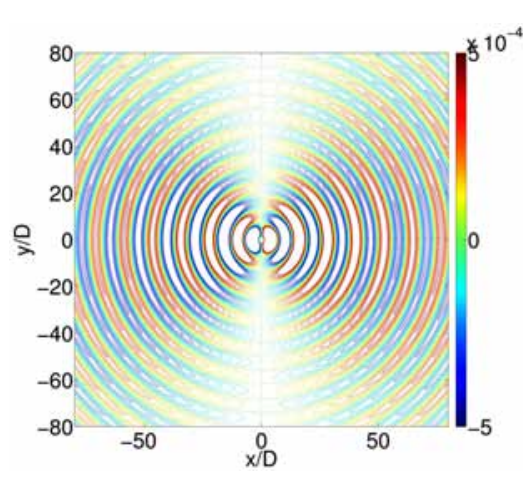

(b) Ellipse

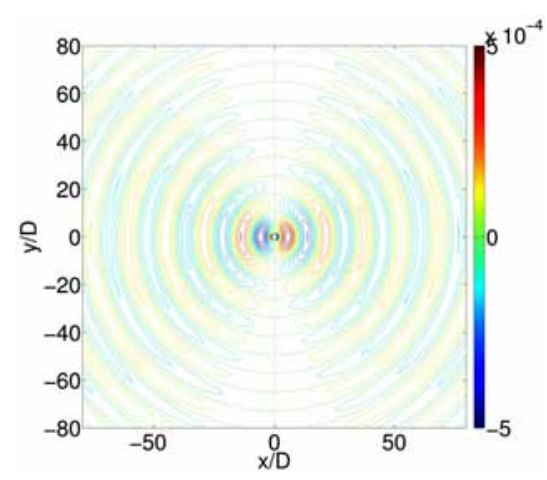

(d) Square

Fig. (9). Contours of sound pressure level due to the fluctuation of drag. 


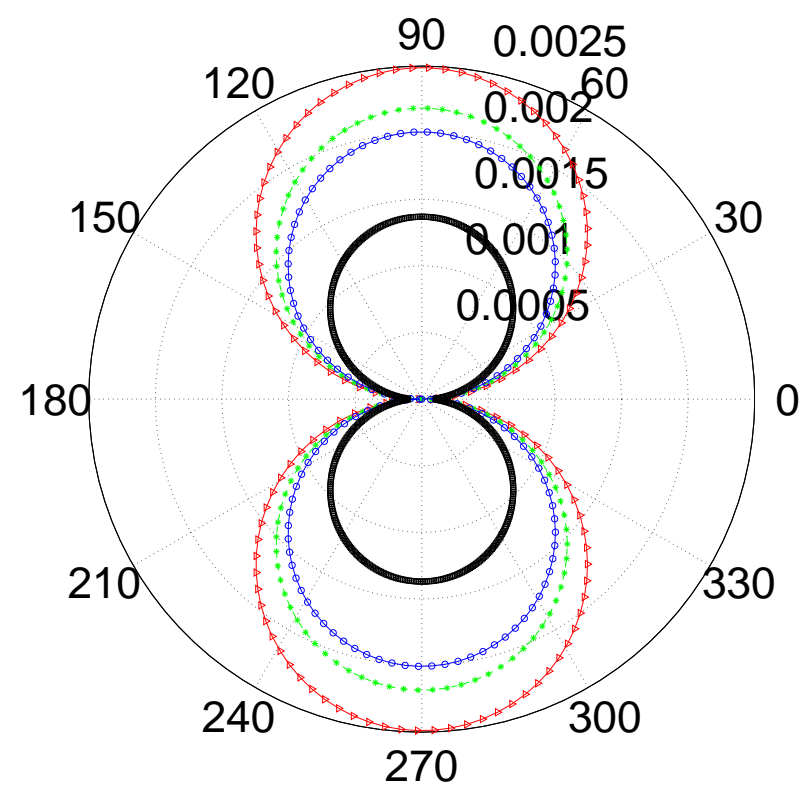

(a) Lift

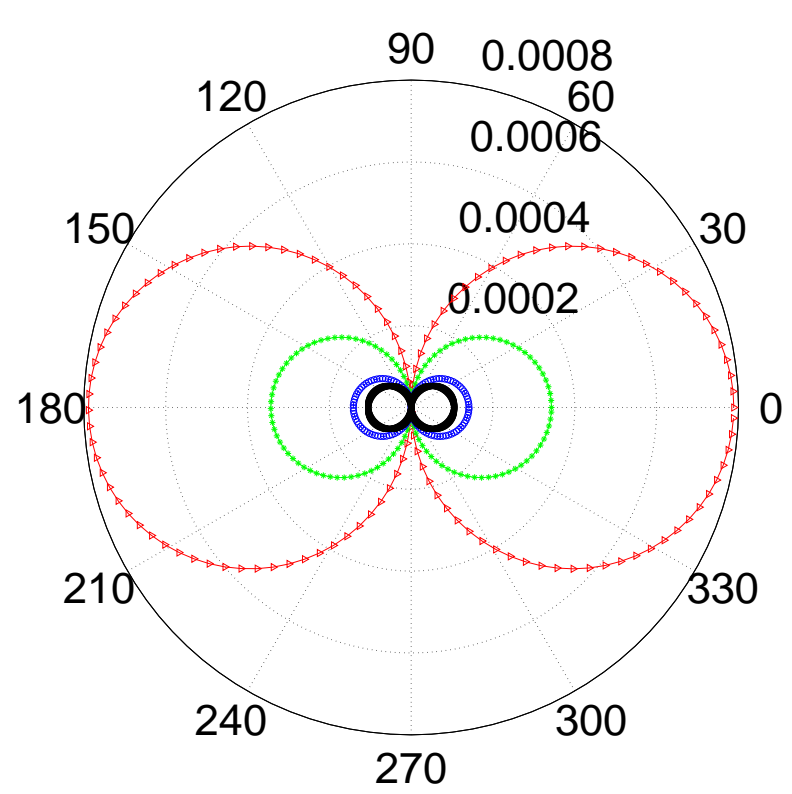

(b) Drag

Fig. (10). Directivity of root mean square sound pressure level ( $\left.\tilde{P}=p_{\mathrm{ms}}^{\prime} / \rho U_{\infty}^{2}\right)$ due to the fluctuation of lift, (a) and drag, (b).

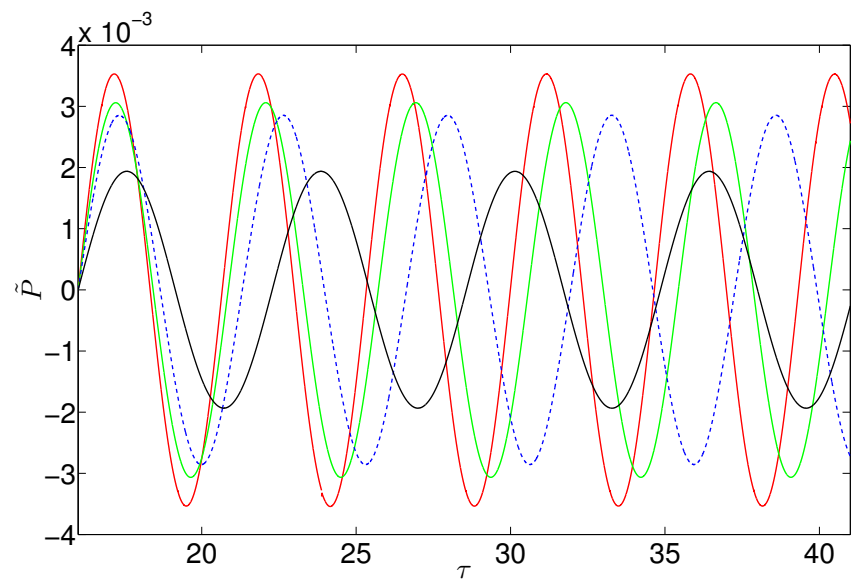

(a) Lift

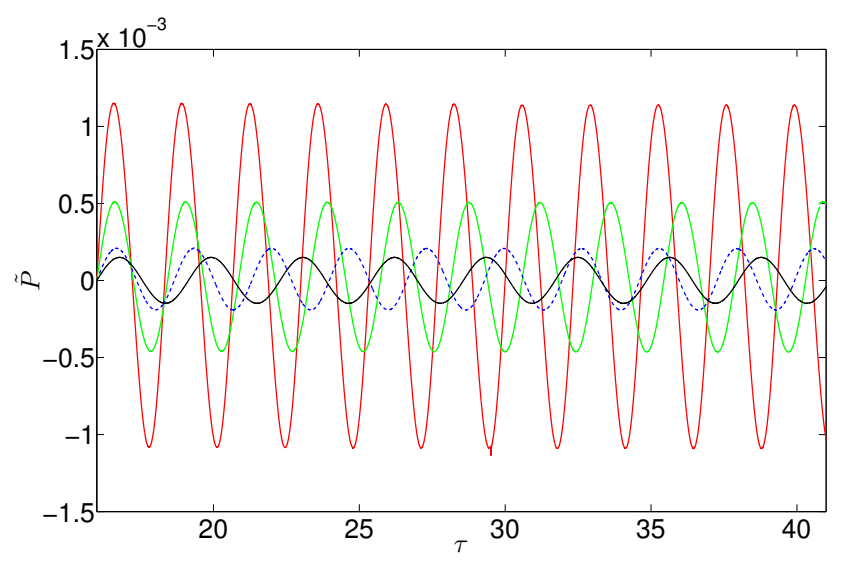

(b) Drag

Fig. (11). Fluctuations of sound pressure due to lift, (a) and drag, (b).

phase. With this current information about the features of sound pressure for various bluff body shapes, a flat plate can now be designed for such a condition. The design will define the suitable gap distance of the flat plate from the upstream body and also the chord length of the flat plate as described in ref. [11].

\section{CONCLUSION}

The flow over various bluff body shapes has been investigated numerically at $R e=150$. The information regarding the flow features and noise generation mechanism has been identified. It was found that all bluff bodies exhibited the same physics features, only the magnitudes were different, in which the triangle cylinder was the highest and square cylinder was the lowest. Inspection on the time histories of the sound pressure level found a sinusoidal pattern in the fluctuation. Therefore, it is possible for a downstream flat plate to be applied in sound cancellation mechanism for all types of the bluff bodies being investigated.

\section{CONFLICT OF INTEREST}

The authors confirm that this article content has no conflict of interest. 


\section{ACKNOWLEDGEMENTS}

The authors would like to acknowledge Universiti Teknologi Malaysia for the receipt of New Academic Staff Grant.

\section{REFERENCES}

[1] K. Ono, R. Himeno, and T. Fukushima, "Prediction of wind noise radiated from passenger cars and its evaluation based on auralization", Journal of Wind Engineering and Industrial Aerodynamics, vol. 81, no. 1-3, pp. 403-419, 1999.

[2] L. S. Hedges, A. K. Travin, and P. R. Spalart, "Detached-eddy simulations over a simplified landing gear", Journal of Fluids Engineering, vol. 124, no. 2, pp. 413-423, 2002.

[3] Y. Li, M. G. Smith, and X. Zhang, "Identification and attenuation of a tonal-noise source on an aircraft's landing gear", Journal of Aircraft, vol. 47, no. 3, pp. 796-804, 2010.

[4] C. Talotte, "Aerodynamic noise: A critical survey", Journal of Sound and Vibration, vol. 231, no. 3, pp. 549-562, 2000.

[5] J. E. Richardson and V. G. Panchang, "Three-dimensional simulation of scour-inducing flow at bridge piers", Journal of Hydraulic Engineering, vol. 124, no. 5, pp. 530-540, 1998.

[6] M. S. M. Ali, C. J. Doolan, and V. Wheatley, "Flow around a square cylinder with a detached downstream flat plate at a low Reynolds number", in 17th Australian Fluid Mechanics Conference, Auckland, New Zealand, 5-9 December 2010

[7] M. S. M. Ali, C. J. Doolan, and V. Wheatley, "Aeolian tones generated by a square cylinder with a splitter plate", in Proceedings of 20th International Congress on Acoustics, ICA 2010, Sydney, Australia, 2327 August 2010.

[8] M. S. M. Ali, C. J. Doolan, and V. Wheatley, "Low Reynolds number flow over a square cylinder with a splitter plate", Physics of Fluids, vol. 23 , no. 3, 2011

[9] M. S. M. Ali, C. J. Doolan, and V. Wheatley, "The sound generated by a square cylinder with a splitter plate at low Reynolds number", Journal of Sound and Vibration, vol. 330, no. 15, pp. 3620-3635, 2011.

[10] M. S. M. Ali, C. J. Doolan, and V. Wheatley, "Low reynolds number flow over a square cylinder with a detached flat plate", International Journal of Heat and Fluid Flow, vol. 36, pp. 22-29, 2012.

[11] M. S. M. Ali, C. J. Doolan, and V. Wheatley, "Aeolian tones generated by a square cylinder with a detached flat plate", AIAA Journal, pp. 1-11, 2013.

[12] C. J. Doolan, "Flat-plate interaction with the near wake of a square cylinder", AIAA Journal, vol. 47, pp. 475-478, 2009.

[13] J. Robichaux, S. Balachandar, and S. P. Vanka, "Three-dimensional floquet instability of the wake of square cylinder", Physics of Fluids, vol. 11, no. 3, pp. 560-578, 1999.

[14] A. K. Saha, G. Biswas, and K. Muralidhar, "Three-dimensional study of flow past a square cylinder at low reynolds numbers", International Journal of Heat and Fluid Flow, vol. 24, pp. 54-66, 2003.

[15] H. Blackburn and J. Lopez, "On three-dimensional quasiperiodic floquet instabilities of two-dimensional bluff body wakes," Physics of Fluids, vol. 15, p. L57, 2003.

[16] S. Luo, X. Tong, and B. Khoo, "Transition phenomena in the wake of a square cylinder", Journal of Fluids and Structures, vol. 23, no. 2, pp. 227-248, 2007

[17] S. C. Luo, Y. T. Chew, and Y. T. Ng, "Characteristics of square cylinder wake transition flows", Physics of Fluids, vol. 15, no. 9, pp. 2549-2559, 2003.

[18] A. Sohankar, C. Norberg, and L. Davidson, "Simulation of threedimensional flow around a square cylinder at moderate reynolds numbers", Physics of Fluids, vol. 11, no. 2, pp. 288-306, 1999.
[19] R. I. Issa, "Solution of the implicitly discretised fluid flow equations by operator-splitting", Journal of Computational physics, vol. 62, no. 1, pp. 40-65, 1986.

[20] H. G. Weller, G. Tabor, H. Jasak, and C. Fureby, "A tensorial approach to computational continum mechanics using object-oriented techniques", Computer in Physics, vol. 12, pp. 620-631, 1998.

[21] M. S. M. Ali, C. J. Doolan, and V. Wheatley, “Grid convergence study for a two-dimensional simulation of flow around a square cylinder at a low reynolds number", Seventh International Conference on CFD in The Minerals and Process Industries, CSIRO (CSIRO Australia, Melbourne, Australia, 2009), 2009.

[22] A. Okajima, "Strouhal numbers of rectangular cylinders", Journal of Fluid Mechanics, vol. 123, pp. 379-398, 1982.

[23] Sohankar, C. Norberg, and L. Davidson, "Low-Reynolds-number flow around a square cylinder at incidence: study of blockage, onset of vortex shedding and outlet boundary condition", International Journal of Numerical Methods in Fluids, vol. 26, no. 1, pp. 39-56, 1998.

[24] O. Inoue, W. Iwakami, and N. Hatakeyama, "Aeolian tones radiated from flow past two square cylinders in a side-by-side arrangement", Physics of Fluids, vol. 18, no. 4, p. 046104, 2006.

[25] M. J. Lighthill, "On sound generated aerodynamically: I. General theory", Proceedings of Royal Society London. Series A, Mathematical and Physical Sciences, vol. 211, no. 1107, pp. 564-587, 1952.

[26] N. Curle, "The influence of solid boundaries upon aerodynamic sound", Proceedings of the Royal Society of London. Series A, Mathematical and Physical Sciences, vol. 231, no. 1187, pp. 505-514, 1955.

[27] O. Inoue and N. Hatakeyama, "Sound generation by a two-dimensional circular cylinder in a uniform flow", Journal of Fluid Mechanics, vol. 471, pp. 285-314, 2002.

[28] M. J. Lighthill, "The bakerian lecture, 1961. Sound generated aerodynamically", Proceedings of the Royal Society of London. Series A. Mathematical and Physical Sciences, vol. 267, no. 1329, pp. 147182, 1962.

[29] D. Crighton, "Basic principles of aerodynamic noise generation", Progress in Aerospace Sciences, vol. 16, no. 1, pp. 31-96, 1975.

[30] J. F. Williams, "Hydrodynamic noise", Annual Review of Fluid Mechanics, vol. 1, no. 1, pp. 197-222, 1969.

[31] J. H. Gerrard, "The mechanics of the formation region of vortices behind bluff bodies", Journal of Fluid Mechanics Digital Archive, vol. 25, no. 02, pp. 401-413, 1966.

[32] A. Roshko, "On the drag and shedding frequency of two-dimensional bluff bodies", NACA Technical Note, no. TN 3169, pp. 1--29, 1954.

[33] O. M. Griffin, "Universal similarity in the wakes of stationary and vibrating bluff structures", Transactions of the ASME, Journal Fluids Engineering, vol. 103, no. 1, 1981.

[34] P. W. Bearman, "Investigation of the flow behind a two-dimensional model with a blunt trailing edge and fitted with splitter plates", Journal of Fluid Mechanics Digital Archive, vol. 21, no. 02,pp. 241-255, 1965.

[35] D. J. J. Leclercq, "Comments on `Aerodynamic noise generation by a stationary body in a turbulent air stream", Journal of Sound and Vibration, vol. 260, no. 1, pp. 183-190, 2003.

[36] M. Farhadi, K. Sedighi, and E. Fattahi, "Effect of a splitter plate on flow over a semi-circular cylinder", Proceedings of the Institution of Mechanical Engineers, Part G: Journal of Aerospace Engineering, vol. 224, no. 3, pp. 321-330, 2010.

[37] S. Mittal, "Computation of three-dimensional flows past circular cylinder of low aspect ratio," Physics of Fluids, vol. 13, no. 1, pp. 177191, 2001.

[38] M. F. Unal and D. Rockwell, "On vortex formation from a cylinder. part 1. the initial instability," Journal of Fluid Mechanics, vol. 190, no. 1, pp. 491-512, 1988.

[39] O. Inoue, M. Mori, and N. Hatakeyama, "Aeolian tones radiated from flow past two square cylinders in tandem", Physics of Fluids, vol. 18, no. 4, p. 046101, 2006. 